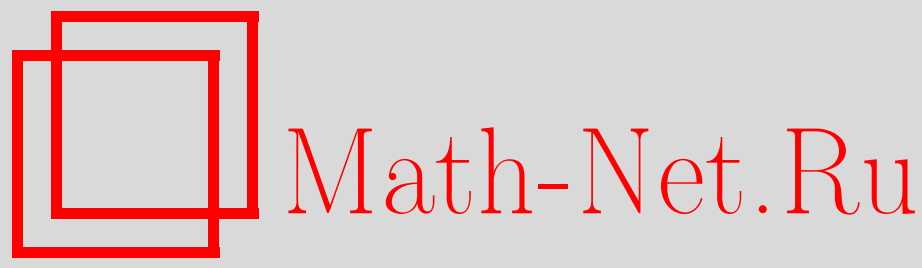

М. О. Корпусов, А. А. Панин, Локальная разрешимость и разрушение решения для уравнения БенджаменаБона-Махони-Бюргерса с нелокальным граничным условием, ТМФ, 2013, том 175, номер 2, 159-172

DOI: https://doi.org/10.4213/tmf8417

Использование Общероссийского математического портала Math-Net.Ru подразумевает, что вы прочитали и согласны с пользовательским соглашением http: //www . mathnet.ru/rus/agreement

Параметры загрузки:

IP : 54.198 .55 .26

26 апреля 2023 г., $11: 43: 28$

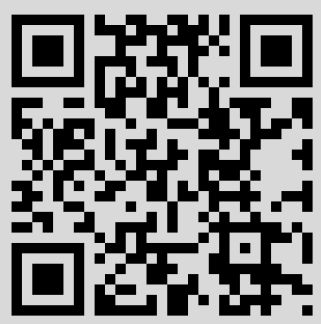




\section{ЛОКАЛЬНАЯ РАЗРЕШИМОСТЬ И РАЗРУШЕНИЕ РЕШЕНИЯ ДЛЯ УРАВНЕНИЯ БЕНДЖАМЕНА-БОНА-МАХОНИ-БЮРГЕРСА С НЕЛОКАЛЬНЫМ ГРАНИЧНЫМ УСЛОВИЕМ}

Рассмотрена модельная начально-краевая задача для уравнения Бенджамена-Бона-Махони-Бюргерса, причем используемые граничные условия имеют физический смысл. Доказана однозначная локальная разрешимость в классическом смысле, получены достаточное условие blowup и оценка на время разрушения. При этом для доказательства blowup использован известный метод пробных функций, развитый в работах В. А. Галактионова, Э. Л. Митидиери и С. И. Похожаева. Заметим, что это один из первых результатов в направлении blowup для рассматриваемого уравнения.

Ключевые слова: blowup, локальная разрешимость, уравнение Бенджамена-Бона-Махони-Бюргерса.

DOI: $10.4213 / \operatorname{tmf} 8417$

\section{1. ВВЕДЕНИЕ}

В 1972 году в классической работе [1] было предложено модельное уравнение для описания длинных волн на воде следующего вида:

$$
u_{x x t}-u_{t}+u_{x x}+\nu\left(u^{2}\right)_{x}=0 .
$$

Однако до настоящего момента, за исключением наших результатов [2], не было получено каких бы то ни было достаточных условий разрушения решения начальной или начально-краевой задачи для этого уравнения. И это при том, что уравнение (1) получило широкое распространение и опубликовано много работ, посвященных исследованию глобальной во времени разрешимости и асимптотического поведения его решений при больших временах [3]-[10].

В настоящей работе мы продолжаем исследования, начатые в работе [2], но теперь мы доказали локальную разрешимость начально-краевой задачи и возникновение blowup. Для доказательства blowup мы пользуемся методом пробных функций [11].

* Московский государственный университет, Москва, Россия.

E-mail: korpusov@rsci.ru, a-panin@yandex.ru 
Отметим, что для получения результатов типа blowup для рассматриваемого уравнения существенно, что граничные условия должны быть не стандартными. Действительно, если мы рассмотрим для уравнения (1) классические граничные условия

$$
u(0, t)=u(l, t)=0,
$$

то несложно показать, что никакого “жесткого" разрушения не будет, поскольку будет иметь место априорная оценка следующего вида:

$$
E(t) \leqslant E(0) e^{-a t}, \quad E(t)=\left\|u_{x}\right\|_{2}^{2}+\|u\|_{2}^{2},
$$

из которой в силу теоремы вложения Соболева получим, что решение задачи для всех $t \in[0,+\infty)$ ограничено. Поэтому для получения blowup нужно рассматривать либо неклассические граничные условия, либо "плохие" классы начальных функций.

\section{2. ВЫВОД УРАВНЕНИЯ}

В силу важности уравнения Бенджамена-Бона-Махони-Бюргерса в физических приложениях мы приведем вывод этого уравнения в теории кристаллических полупроводников (см. [12]) и рассмотрим физический смысл используемых в следующем разделе граничных условий.

Итак, рассмотрим электрическую часть системы уравнений Максвелла в квазистационарном приближении в некоторой области $\Omega \subset \mathbb{R}^{3}$, имеющей поверхностно односвязную границу $\partial \Omega$ :

$$
\operatorname{div} \mathbf{D}=-n, \quad \mathbf{D}=\mathbf{E}+\mathbf{P}, \quad \operatorname{rot} \mathbf{E}=0,
$$

где $\mathbf{D}$ - вектор индукции электрического поля, $\mathbf{E}$ - вектор напряженности электрического поля, $\mathbf{P}$ - вектор поляризации среды, $n$ - плотность свободных зарядов. Теперь заметим, что по своему физическому смыслу величина

$$
\operatorname{div} \mathbf{P}=\rho
$$

есть плотность так называемых связанных зарядов. Рассмотрим теперь кристаллический полупроводник, помещенный во внешнее постоянное электрическое поле $\mathbf{E}_{0}$, причем выберем ортогональный репер $\left\{\mathbf{e}_{1}, \mathbf{e}_{2}, \mathbf{e}_{3}\right\}$ декартовой системы координат таким образом, чтобы

$$
\mathbf{E}_{0}=E_{0} \mathbf{e}_{1}
$$

В кристаллическом полупроводнике как в проводящей среде имеет место следующее уравнение:

$$
\frac{\partial n}{\partial t}=\operatorname{div} \mathbf{J}
$$

где $\mathbf{J}$ - вектор тока свободных зарядов. Справедливо следующее соотношение, связывающее этот вектор с вектором $\mathbf{E}$ напряженности электрического поля:

$$
\mathbf{J}=\sigma \mathbf{E}+e h(\phi) \mathbf{E}_{0}, \quad \mathbf{E}=-\nabla \phi, \quad \sigma>0,
$$

где функция $h(\phi)$ описывает квазистационарное распределение плотности свободных зарядов, поэтому эта величина должна быть неотрицательной, множитель $e-$ 
заряд электрона. Рассмотрим теперь явный вид функции $h(\phi)$ из (6) и функции $\rho(\phi)$ из формулы (3). Действительно, рассмотрим случай, когда

$$
h(\phi)=h_{0} \phi^{2}, \quad \rho=\rho_{0} \phi, \quad h_{0}>0, \quad \rho_{0}>0 .
$$

Дифференциальным следствием системы уравнений (2)-(7) является уравнение

$$
\frac{\partial}{\partial t}\left(\triangle \phi-\rho_{0} \phi\right)-\sigma \triangle \phi+e h_{0} E_{0} \frac{\partial}{\partial x_{1}} \phi^{2}=0,
$$

которое легко сводится к следующему виду:

$$
\frac{\partial}{\partial t}(\triangle \phi-\phi)+\triangle \phi+\mu \frac{\partial}{\partial x_{1}} \phi^{2}=0, \quad x=\left(x_{1}, x_{2}, x_{3}\right) \in \Omega \subset \mathbb{R}^{3},
$$

причем $\mu>0$. Обсудим теперь различные виды граничных условий. Прежде всего, условие

$$
\left.\phi\right|_{\partial \Omega}=0
$$

означает, что граница полупроводника $\partial \Omega$ заземлена, а по договоренности электрический потенциал земли считается равным нулю. Поскольку $\mathbf{E}=-\nabla \phi$, то условие

$$
\left.\frac{\partial \phi}{\partial n_{x}}\right|_{\partial \Omega}=-\left.\left(\mathbf{E}, \mathbf{n}_{x}\right)\right|_{\partial \Omega}
$$

означает задание нормальной компоненты электрического поля на границе, что естественно.

Отметим, что физический смысл разрушения решения уравнения (8) - это экспериментально наблюдаемый электрический "пробой" в кристаллическом полупроводнике.

Предположим теперь, что область $\Omega$ - это слой $\Pi=\left\{x \in[0, l],(y, z) \in \mathbb{R}^{2}\right\}$. Тогда, во-первых, при начальном условии

$$
\phi(x, y, z, 0)=\phi_{0}(x, y, z),
$$

таком, что функция $\phi_{0}(x, y, z)$ зависит только от $x \in[0, l]$, мы можем редуцировать нашу задачу к одномерной задаче на указанном отрезке. Кроме того, мы рассмотрим следующее нелокальное условие:

$$
\left.\frac{\partial \phi(x, y, z)}{\partial x}\right|_{x=0}=\frac{1}{l} \int_{0}^{l} \frac{\partial \phi}{\partial x} d x=\frac{\phi(l, y, z, t)-\phi(0, y, z, t)}{l},
$$

которое означает, что величина $x$-компоненты электрического поля на левой границе слоя равна средней величине этой компоненты по всему слою. При этом если плоскость $x=0$ заземлена, то имеет место граничное условие

$$
\left.\phi(x, y, z, t)\right|_{x=0}=0 .
$$

Тогда граничное условие (9) примет вид

$$
\frac{\partial \phi(0, y, z, t)}{\partial x}=\frac{\phi(l, y, z, t)}{l} .
$$

Таким образом, граничные условия (10) и (11) имеют физический смысл. 


\section{3. ЛОКАЛЬНАЯ РАЗРЕШИМОСТЬ}

3.1. Теорема о непродолжаемом решении. Мы будем последовательно использовать следующую теорему.

Теорема 1. Пусть рассматривается абстрактная задача Коши

$$
y^{\prime}(t)=A(t)(y), \quad y(0)=y_{0}
$$

в банаховом пространстве $B$ с нормой $\|\cdot\|$. Под решением задачи Коши (12) понимается функция $y \in C^{1}\left(\left[0, T_{0}\right), B\right)$, где $T_{0}<+\infty$ или $T_{0}=+\infty$, а уравнение понимается в смысле сильного дифференцирования в банаховом пространстве $B$ при всех $t \in\left[0, T_{0}\right)$. Пусть $y_{0} \in B$ и пусть (нелинейный) оператор $A(t)(y)$, зависящий от числового параметра $t \geqslant 0$, удовлетворяет следующим условиям:

1) $A(t)(\theta)=\theta$ при всех $t \geqslant 0(\theta$ - нулевой элемент пространства $B)$;

2 ) оператор $A(t)(y)$ локально липшич-непрерьвен равномерно по $t \geqslant 0$, т.е. для любых $z_{1}, z_{2} \in B$ и любого $t \geqslant 0$ выполнено неравенство

$$
\left\|A(t)\left(z_{1}\right)-A(t)\left(z_{2}\right)\right\| \leqslant \mu\left(\max \left(\left\|z_{1}\right\|,\left\|z_{2}\right\|\right)\right)\left\|z_{1}-z_{2}\right\|
$$

где функиия $\mu:[0,+\infty) \rightarrow[0,+\infty)$ ограничена на каждом ограниченном подножестве луча $[0,+\infty)$;

3) оператор $A(t)(y)$ непрерывен по совокупности переменных $(t, y)$.

Тогда существует и единственно решение, для которого или $T_{0}=+\infty$, или $T_{0}<+\infty$, причем в последнем случае выполняется предельное соотношение

$$
\underset{t \uparrow T_{0}}{\limsup }\|A(t)(y(t))\|=+\infty
$$

Такое решение мы будем называть непродолжаемым, а любое другое решение может быть продолжено до решения, удовлетворяющего указанному условию. Иными словами, любое другое решение есть не что иное, как ограничение непродолжаемого решения на менъший временной промежуток.

Чтобы не разрывать изложение основного материала работы, доказательство теоремы 1 вынесено в приложение.

3.2. Постановка задачи и ее эквивалентные переформулировки. Итак, рассматривается начально-краевая задача

$$
\begin{gathered}
\frac{\partial}{\partial t}\left(u_{x x}-u\right)+u_{x x}+u u_{x}=0, \quad(x, t) \in[0, l] \times\left[0, T_{0}\right), \\
u(x, 0)=u_{0}(x), \quad x \in[0, l], \\
u(0, t)=0, \quad l u_{x}(0, t)=u(l, t), \quad t \in\left[0, T_{0}\right),
\end{gathered}
$$

где $u_{0}(x) \in C^{2}([0, l])$ и удовлетворяет граничным условиям (17). Величина $T_{0}$, которая может быть конечной или бесконечной $\left(T_{0}=+\infty\right)$, будет определена ниже. Здесь и далее производные по переменной $x$ обозначены нижним индексом (даже для функций, зависящих только от $x$ ), а штрих может обозначать лишь производную по времени $t$. 
Нам потребуется ввести функциональные пространства

$$
\begin{gathered}
Z=C([0, l]), \quad\|z\|_{Z}=\|z\|_{C([0, l])} \\
Z_{1}=\left\{z(x) \in C^{1}([0, l]) \mid z(0)=0, l z_{x}(0)=z(l)\right\}, \quad\|z\|_{Z_{1}}=\|z\|_{C([0, l])}+\left\|z_{x}\right\|_{C([0, l])}, \\
Z_{2}=\left\{z(x) \in C^{2}([0, l]) \mid z(0)=0, l z_{x}(0)=z(l)\right\} \\
\|z\|_{Z_{2}}=\|z\|_{C([0, l])}+\left\|z_{x}\right\|_{C([0, l])}+\left\|z_{x x}\right\|_{C([0, l])} .
\end{gathered}
$$

Пространства $Z_{1}, Z_{2}$, очевидно, полны относительно выбранных норм как замкнутые подпространства пространств $C^{1}([0, l])$ и $C^{2}([0, l])$ соответственно.

Введем также непрерывный оператор $\mathbb{L}: Z_{2} \rightarrow Z$ по формуле

$$
\mathbb{L} z=z_{x x}-z .
$$

Оператор $\mathbb{L}$ имеет непрерывный обратный оператор $\mathbb{G}: Z \rightarrow Z_{2}$, который можно выписать явно:

$$
(\mathbb{G} f)(x)=\int_{0}^{l} G(x, s) f(s) d s,
$$

с помощью функции Грина задачи

$$
\begin{aligned}
v_{x x}-v & =f(x), \quad x \in[0, l], \\
v(0) & =0 \\
l v_{x}(0) & =v(l) .
\end{aligned}
$$

Эта функция Грина, как нетрудно проверить непосредственно, имеет вид

$$
G(x, s)=G_{0}(x, s)+\frac{\operatorname{sh} x(l \operatorname{ch} s-\operatorname{ch} l \operatorname{sh} s)}{l-\operatorname{sh} l},
$$

где

$$
G_{0}(x, s)= \begin{cases}-\operatorname{sh} x \operatorname{ch} s, & 0 \leqslant x \leqslant s \leqslant l, \\ -\operatorname{ch} x \operatorname{sh} s, & 0 \leqslant s \leqslant x \leqslant l .\end{cases}
$$

Непрерывность оператора $\mathbb{G}: Z \rightarrow Z_{2}$ следует из общих свойств функции Грина, а также может быть легко проверена непосредственно для явно выписанной функции Грина.

ОПРЕДЕЛЕниЕ 1. Решением задачи (15)-(17) будем называть функцию

$$
u(x)(t) \in C^{1}\left(\left[0, T_{0}\right), Z_{2}\right),
$$

где $T_{0}<+\infty$ или $T_{0}=+\infty$, удовлетворяющую в классическом смысле уравнению (15) и начальному условию (16) (отметим, что граничные условия включены в определения пространств $Z_{1}, Z_{2}$ ). Легко видеть, что в силу гладкости решения (19) входящие в уравнение производные существуют и непрерывны. Более того, из (19) следует, что операторы $\partial^{2} / \partial x^{2}$ и $\partial / \partial t$ коммутируют, поэтому уравнение (15) может быть переписано в виде

$$
\mathbb{L} u^{\prime}+u_{x x}+u u_{x}=0
$$


где производную по времени можно считать обычной (не частной) в смысле пространства (19). Далее, уравнение $(20)$ в силу обратимости операторов $\mathbb{L}=\mathbb{G}^{-1}$ эквивалентно в пространстве (19) уравнению

$$
u^{\prime}+\mathbb{G}\left(u_{x x}\right)+\mathbb{G}\left(u u_{x}\right)=0 .
$$

Поскольку $\mathbb{G}\left(u_{x x}\right)=\mathbb{G}\left(u_{x x}-u+u\right)=u+\mathbb{G} u$, мы приходим к эквивалентному виду

$$
u^{\prime}+u+\mathbb{G} u+\mathbb{G}\left(u u_{x}\right)=0 .
$$

Сделав теперь в (21) замену

$$
w=u e^{t},
$$

мы получим в силу сказанного выше, что функция $u(x)(t) \in C^{1}\left(\left[0, T_{0}\right), Z_{2}\right)$ является решением задачи (15)-(17) тогда и только тогда, когда функция $w(x)(t) \in$ $C^{1}\left(\left[0, T_{0}\right), Z_{2}\right)$, связанная с ней тождеством $(22)$, является решением задачи Коши

$$
w^{\prime}=-\left(\mathbb{G} w+e^{-t} \mathbb{G}\left(w w_{x}\right)\right), \quad w(x)(0)=u_{0}(x) \equiv w_{0}(x) \in Z_{2} .
$$

Стандартным образом задача (23) может быть сведена к интегральному уравнению

$$
w(t)=w_{0}-\int_{0}^{t} d \tau A(\tau)(w(\tau)),
$$

где $w_{0}=u_{0}(x), A(t)(z)=\mathbb{G} z+e^{-t} \mathbb{G}\left(z z_{x}\right)$.

3.3. Интегральное уравнение в пространстве $C^{1}\left(\left[0, T_{0}\right), Z_{1}\right)$. Будем вначале искать решение интегрального уравнения (24) более ослабленного типа, т. е. при $w(x)(t) \in C^{1}\left(\left[0, T_{0}\right), Z_{1}\right)$. Отметим, что по-прежнему $w_{0} \in Z_{2} \subset Z_{1}$.

Нетрудно видеть, что оператор

$$
A(t)(z): z \mapsto \mathbb{G} z+e^{-t} \mathbb{G}\left(z z_{x}\right)
$$

локально липшиц-непрерывен как отображение $Z_{1} \rightarrow Z_{1}$ в силу свойств функции Грина. (Ниже будет доказано более сильное утверждение.) Далее, этот оператор непрерывен и по совокупности переменных $(t, z)$ в силу непрерывности произведения непрерывных функций $e^{-t}: \mathbb{R} \rightarrow \mathbb{R}$ и $\mathbb{G}\left(z z_{x}\right): Z_{1} \rightarrow Z_{1}$. Кроме того, $A(t)(0)=0$. Итак, оператор $A(t)(z)$ удовлетворяет всем условиям теоремы 1. Поэтому в силу теоремы 1 уравнение (24) имеет единственное непродолжаемое решение. Точнее, верна

Теорема 2. Решение интегрального уравнения (24) (или, что то же самое, задачи Коши (23)) существует на некотором максимальном промежутке $\left[0, T_{0}\right)$, где $0<T_{0} \leqslant+\infty$, и единственно на нем. Более того, если $T_{0}<+\infty$, то имеет место равенство

$$
\limsup _{t \uparrow T_{0}}\|A w\|_{Z_{1}}=+\infty
$$




\section{4. Повышение гладкости до $C^{1}\left(\left[0, T_{0}\right), Z_{2}\right)$.}

Теорема 3. Пусть на промежутке $\left[0, T_{0}\right)$ (әде $T_{0}$ может быть конечным или бесконечным) существует решение $w(x)(t) \in C^{1}\left(\left[0, T_{0}\right), Z_{1}\right)$ задачи Коши (23) (или, что то же самое, интегрального уравнения (24)). Тогда это решение принадлежит классу $C^{1}\left(\left[0, T_{0}\right), Z_{2}\right)$.

ДокАЗАТЕЛЬСтво. Заметим, что оператор

$$
A(t)(z): z \mapsto \mathbb{G} z+e^{-t} \mathbb{G}\left(z z_{x}\right)
$$

локально липшиц-непрерывен как отображение $Z_{1} \rightarrow Z_{2}$ с не зависящей от $t$ константой Липшица. Действительно, имеем при всех $t \geqslant 0$

$$
\begin{aligned}
\|A(t)(\bar{z})-A(t)(\overline{\bar{z}})\|_{Z_{2}} & =\left\|\mathbb{G}(\bar{z}-\overline{\bar{z}})+e^{-t} \mathbb{G}\left(\bar{z} \bar{z}_{x}\right)-e^{-t} \mathbb{G}\left(\overline{\bar{z}} \overline{\bar{z}}_{x}\right)\right\|_{Z_{2}} \leqslant \\
& \leqslant\|\mathbb{G}\|_{Z \rightarrow Z_{2}}\|\bar{z}-\overline{\bar{z}}\|_{Z}+e^{-t}\left\|\mathbb{G}\left(\bar{z} \bar{z}_{x}\right)-\mathbb{G}\left(\overline{\bar{z}} \overline{\bar{z}}_{x}\right)\right\|_{Z_{2}} \leqslant \\
& \leqslant\|\mathbb{G}\|_{Z \rightarrow Z_{2}}\|\bar{z}-\overline{\bar{z}}\|_{Z_{1}}+\left\|\mathbb{G}\left(\bar{z} \bar{z}_{x}-\overline{\bar{z}} \overline{\bar{z}}_{x}\right)\right\|_{Z_{2}} .
\end{aligned}
$$

Для оценки второго слагаемого в правой части заметим, что

$$
\begin{aligned}
\left\|\bar{z} \bar{z}_{x}-\overline{\bar{z}} \overline{\bar{z}}_{x}\right\|_{Z} & =\left\|\bar{z} \bar{z}_{x}-\bar{z} \overline{\bar{z}}_{x}+\bar{z} \overline{\bar{z}}_{x}-\overline{\bar{z}} \overline{\bar{z}}_{x}\right\|_{Z} \leqslant\left\|\bar{z}\left(\bar{z}_{x}-\overline{\bar{z}}_{x}\right)\right\|_{Z}+\left\|(\bar{z}-\overline{\bar{z}}) \overline{\bar{z}}_{x}\right\|_{Z} \leqslant \\
& \leqslant\|\bar{z}\|_{Z}\|\bar{z}-\overline{\bar{z}}\|_{Z_{1}}+\|\bar{z}-\overline{\bar{z}}\|_{Z}\|\overline{\bar{z}}\|_{Z_{1}} \leqslant \\
& \leqslant\|\bar{z}\|_{Z_{1}}\|\bar{z}-\overline{\bar{z}}\|_{Z_{1}}+\|\bar{z}-\overline{\bar{z}}\|_{Z_{1}}\|\overline{\bar{z}}\|_{Z_{1}} \leqslant 2 \max \left(\|\bar{z}\|_{Z_{1}},\|\overline{\bar{z}}\|_{Z_{1}}\right)\|\bar{z}-\overline{\bar{z}}\|_{Z_{1}} .
\end{aligned}
$$

Поэтому в силу непрерывности линейного оператора $\mathbb{G}$ как отображения $Z \rightarrow Z_{2}$ мы получаем требуемый результат с константой Липшица, зависящей от $\max \left(\|\bar{z}\|_{Z_{1}},\|\overline{\bar{z}}\|_{Z_{1}}\right)$.

Итак, $A(t)$ есть локально липшиц-непрерывный оператор как отображение из $Z_{1}$ в $Z_{2}$, причем константа Липшица зависит от $\max \left(\|\bar{z}\|_{Z_{1}},\|\overline{\bar{z}}\|_{Z_{1}}\right)$ и не зависит от $t$. Отметим, что локальная липшиц-непрерывность оператора $A(t)(z): Z_{1} \rightarrow Z_{1}$ отсюда непосредственно следует в силу непрерывности вложения $Z_{2} \rightarrow Z_{1}$ с константой вложения, не превышающей единицу для выбранных нормировок пространств $Z_{1}$ и $Z_{2}$. Из только что доказанной локальной липшиц-непрерывности с учетом очевидного равенства $A(t)(0)=0$ имеем при всех $t \geqslant 0$

$$
\|A(t)(z)\|_{Z_{2}} \leqslant L\left(\|z\|_{Z_{1}}\right)\|z\|_{Z_{1}}
$$

где вещественная функция $L(\cdot)$ ограничена на каждом ограниченном подмножестве луча $[0,+\infty)$. Далее, оператор $A(t)(z)$ непрерывен по совокупности переменных $(t, z)$ в силу непрерывности произведения непрерывных функций $e^{-t}: \mathbb{R} \rightarrow \mathbb{R}$ и $\mathbb{G}\left(z z_{x}\right)$ : $Z_{1} \rightarrow Z_{2}$. Следовательно, в силу того что композиция непрерывных отображений непрерывна, мы получаем, что если $w(x)(t) \in C^{1}\left(\left[0, T_{0}\right), Z_{1}\right) \subset C\left(\left[0, T_{0}, Z_{1}\right)\right)$, то $A(t)(w(x)(t)) \in C\left(\left[0, T_{0}\right), Z_{2}\right)$, поэтому интеграл в правой части уравнения $(24)$ существует и в смысле пространства $Z_{2}$ и

$$
\left\|\int_{0}^{t} A(\tau) w(\tau) d \tau\right\|_{Z_{2}} \leqslant \int_{0}^{t}\|A(\tau) w(\tau)\|_{Z_{2}} d \tau \leqslant \int_{0}^{t} L\left(\|w(\tau)\|_{Z_{1}}\right)\|w(\tau)\|_{Z_{1}} d \tau,
$$

где в силу (25) функция $L\left(\|w(\tau)\|_{Z_{1}}\right)$ ограничена на $[0, t]$ при любом $t<T_{0}$. Следовательно, правая часть уравнения (24) принадлежит $Z_{2}$ при каждом $t$ (напомним, 
что $\left.w_{0} \in Z_{2}\right)$ и дифференцируема как функция от $t$ со значениями в $Z_{2}$, являясь интегралом от непрерывной функции. То же можно сказать о левой части, и мы заключаем, что $w(x)(t) \in C^{1}\left(\left[0, T_{0}\right), Z_{2}\right)$.

Теорема доказана.

3.5. Некоторые более сильные результаты. Можно, однако, исходить из решения не в пространстве $Z_{1}$, а в пространстве $Z$. Для этого нам следует распространить оператор $A(t)(z)$ на функции $z \in C([0, l])$. Для этого рассмотрим более подробно оператор $\mathbb{G}$ (см. (18) и ниже). Положим

$$
\begin{aligned}
& g_{1}(x, s)=-\operatorname{sh} x \operatorname{ch} s+\frac{\operatorname{sh} x(l \operatorname{ch} s-\operatorname{ch} l \operatorname{sh} s)}{l-\operatorname{sh} l}, \\
& g_{2}(x, s)=-\operatorname{ch} x \operatorname{sh} s+\frac{\operatorname{sh} x(l \operatorname{ch} s-\operatorname{ch} l \operatorname{sh} s)}{l-\operatorname{sh} l} .
\end{aligned}
$$

Тогда

$$
(\mathbb{G} f)(x)=\int_{0}^{x} g_{2}(x, s) f(s) d s+\int_{x}^{l} g_{1}(x, s) f(s) d s .
$$

Естественно, это определение не годится для функции $f=z z_{x}$, если $z$ только непрерывна. Поэтому мы формально запишем $z z_{x}=(1 / 2)\left(z^{2}\right)_{x}$ и формально проинтегрируем по частям в (27). Это даст

$$
\begin{aligned}
\mathbb{G}\left(z z_{x}\right)(x)=\frac{1}{2} & {\left[\int_{0}^{x} g_{2}(x, s)\left(z^{2}(s)\right)_{s} d s+\int_{x}^{l} g_{1}(x, s)\left(z^{2}(s)\right)_{s} d s\right]=} \\
=\frac{1}{2} & {\left[\left.g_{2}(x, s) z^{2}(s)\right|_{s=0} ^{s=x}-\right.} \\
& \left.\quad-\int_{0}^{x} \frac{\partial g_{2}}{\partial s} z^{2}(s) d s+\left.g_{1}(x, s) z^{2}(s)\right|_{s=x} ^{s=l}-\int_{x}^{l} \frac{\partial g_{1}}{\partial s} z^{2}(s) d s\right]= \\
=\frac{1}{2} & {\left[g_{1}(x, l) z^{2}(l)-g_{2}(x, 0) z^{2}(0)-\int_{0}^{x} \frac{\partial g_{2}}{\partial s} z^{2}(s) d s-\int_{x}^{l} \frac{\partial g_{1}}{\partial s} z^{2}(s) d s\right], }
\end{aligned}
$$

где в последнем равенстве мы воспользовались непрерывностью функции Грина. Формулу (28) следует рассматривать как определение оператора $\mathbb{G}\left(z z_{x}\right)$, применимое к $z \in Z$. Существенно, однако, что при $z \in Z_{1}$ цепочку равенств (28) можно прочитать с конца и убедиться тем самым, что при таких $z$ новое определение равносильно старому. Далее мы предположим, что второе слагаемое в операторе $A(t)(z)$ определено с помощью формулы (28).

Заметим теперь, что функция $\left(\mathbb{G}\left(z z_{x}\right)\right)(x)$ дифференцируема по $x$, как следует из свойств интегралов, зависящих от параметров, и свойств функции Грина. Имеем

$$
\begin{aligned}
\left(\mathbb{G}\left(z z_{x}\right)\right)_{x}(x)= & \frac{1}{2}\left[\frac{\partial g_{1}}{\partial x}(x, l) z^{2}(l)-\frac{\partial g_{2}}{\partial x}(x, 0) z^{2}(0)-\left.\frac{\partial g_{2}}{\partial s}(x, s)\right|_{s=x} z^{2}(x)-\right. \\
& -\int_{0}^{x} \frac{\partial^{2} g_{2}}{\partial x \partial s}(x, s) z^{2}(s) d s+\left.\frac{\partial g_{1}}{\partial s}(x, s)\right|_{s=x} z^{2}(x)- \\
& \left.-\int_{x}^{l} \frac{\partial^{2} g_{2}}{\partial x \partial s}(x, s) z^{2}(s) d s\right] .
\end{aligned}
$$


Кроме того, отметим, что при $z \in Z$ для функции $A(t)(z)$ выполнены граничные условия (17), это следует из свойств функции Грина. Поэтому при $z \in Z$ имеем $A(t)(z) \in Z_{1}$.

Используя ограниченность функций $g_{1}(x, s)$ и $g_{2}(x, s)$ и их первых и вторых производных на отрезке $[0, l]$, а также неравенство

$$
\left|z_{1}^{2}(x)-z_{2}^{2}(x)\right|=\left|z_{1}(x)-z_{2}(x)\right| \cdot\left|z_{1}(x)+z_{2}(x)\right| \leqslant\left\|z_{1}-z_{2}\right\|_{C([0, l])} \cdot\left(\left\|z_{1}\right\|_{C([0, l])}+\left\|z_{2}\right\|_{C([0, l])}\right) \text {, }
$$

устанавливаем, что оператор $z \mapsto \mathbb{G}\left(z z_{x}\right)$, а с ним и оператор $A(t)(z)$ (в котором второе слагаемое теперь определено формулой (28)), является равномерно по $t$ локально липшиц-непрерывным как отображение $Z \rightarrow Z_{1}$. А из теоремы о непрерывности произведения получаем, как и прежде, что оператор $A(t)(z)$ непрерывен по совокупности переменных $(t, z)$ относительно рассматриваемых норм. Это также верно, если рассматривать оператор $A$ как отображение $Z \rightarrow Z$. Итак, для оператора $A(t)(z)$ в указанном смысле выполнены все условия теоремы 1 , и, следовательно, уравнение (24) имеет единственное непродолжаемое решение $w(x)(t)$ в классе

$$
C^{1}\left(\left[0, T_{0}\right), Z\right)
$$

Далее, пользуясь только что установленными равномерной по $t$ локальной липшиц-непрерывностью и непрерывностью по совокупности переменных $(t, z)$ оператора $A(t)(z)$ как отображение $Z \rightarrow Z_{1}$, как и в предыдущем разделе, получаем, что существование решения из класса $(30)$ на промежутке $\left[0, T_{0}\right)$ гарантирует существование решения из класса $w(x)(t) \in C^{1}\left(\left[0, T_{0}\right), Z_{1}\right)$ на этом же промежутке. А последнее в силу теоремы 3 гарантирует существование классического решения $w(x)(t) \in C^{1}\left(\left[0, T_{0}\right), Z_{2}\right)$ на этом же промежутке. Эти рассуждения доказывают, что верна

Теорема 4. 1. Решение интегралъного уравнения (24) (или, что то же самое, задачи Коши (23)) в классе (30) существует на некотором максимальном промежутке $\left[0, T_{0}\right)$, где $0<T_{0} \leqslant+\infty$, и единственно на нем. Более того, равенство

$$
\limsup _{t \uparrow T_{0}}\|A w\|_{Z}=+\infty
$$

имеет место при $T_{0}<+\infty$.

2. Существование решения интегрального уравнения (24) в классе (30) гарантирует существование его решения в классе $C^{1}\left(\left[0, T_{0}\right), Z_{2}\right)$ с тем же $T_{0}$.

\section{4. РАЗРУШЕНИЕ РЕШЕНИЯ}

В предыдущем разделе мы доказали существование единственного непродолжаемого решения задачи (15)-(17), понимаемого в классическом смысле

$$
u(x)(t) \in C^{1}\left(\left[0, T_{0}\right), C^{2}([0, l])\right) .
$$

Теперь умножим обе части уравнения (15) на пробную функцию $l-x$ и проинтегрируем по $x \in(0, l)$. С учетом граничных условий с помощью интегрирования по 
частям получаем

$$
\begin{aligned}
& \int_{0}^{l}(l-x) u_{x x}^{\prime} d x=-l u_{x}^{\prime}(0, t)+u^{\prime}(l, t)-u^{\prime}(0, t)=0 \\
& \int_{0}^{l}(l-x) u_{x x} d x=-l u_{x}(0, t)+u(l, t)-u(0, t)=0 \\
& \int_{0}^{l}(l-x) u u_{x} d x=\frac{1}{2} \int_{0}^{l} u^{2}(x, t) d x
\end{aligned}
$$

Таким образом, с учетом уравнения (15) приходим к следующему равенству:

$$
\frac{d J}{d t}=\frac{1}{2} \int_{0}^{l} u^{2} d x, \quad J(t)=\int_{0}^{l}(l-x) u d x
$$

Справедлива цепочка неравенств

$$
\frac{d J}{d t} \geqslant \frac{1}{2} \int_{0}^{l} \frac{l-x}{l} u^{2} d x=\frac{1}{2 l} \frac{l^{2}}{2} \int_{0}^{l} \frac{l-x}{l^{2} / 2} u^{2} d x \geqslant \frac{1}{2 l} \frac{l^{2}}{2}\left(\int_{0}^{l} \frac{l-x}{l^{2} / 2} u d x\right)^{2}=\frac{1}{l^{3}} J^{2}(t),
$$

где мы воспользовались неравенством Йенсена [13] для выпуклых функций. Теперь мы предположим, что начальная функция $u_{0}(x)$ удовлетворяет условию

$$
J(0)=\int_{0}^{l}(l-x) u_{0}(x) d x>0
$$

Тогда из (32) получим неравенство

$$
J(t) \geqslant \frac{J(0)}{1-l^{-3} J(0) t}
$$

из которого следует, что

$$
T_{0} \leqslant T_{1} \equiv l^{3} J(0)^{-1}
$$

(т. е. на большем временном промежутке решение существовать не может) и, кроме того, в случае существования решения на промежутке $\left[0, T_{1}\right)$ с $T_{1}=l^{3} J(0)^{-1}$ имеет место предельное свойство

$$
\lim _{T \uparrow T_{1}}\|u(x)(t)\|_{C([0, l])}=+\infty
$$

Итак, мы доказали следующую теорему.

Теорема 5. При условии (33) задача (15)-(17) не имеет решения в классе (19): интервал существования [0, $\left.T_{0}\right)$ ограничен условием (34). 


\section{5. ОСНОВНОЙ РЕЗУЛЬТАТ}

Из изложенных выше результатов непосредственно следует

Теорема 6. В условиях теорем 2, 5 решение задачи (15)-(17) существует в классическом смысле (31) и разрушается за конечное время с режимом "жесткий blowup", m.e. $T_{0} \leqslant T_{1} \equiv l^{3} J(0)^{-1} u$

$$
\limsup _{t \uparrow T_{0}}\|u(x)(t)\|_{C([0, l])}=+\infty .
$$

ДокАЗАтЕЛьство. В самом деле, теорема 4 с учетом замены (22) гарантирует существование и единственность решения в классическом смысле, причем решение принадлежит $C^{1}\left(\left[0, T_{0}\right), C^{2}([0, l])\right)$ для тех же самых $T_{0}$, для которых оно принадлежит $C^{1}\left(\left[0, T_{0}\right), C([0, l])\right)$. Теорема 5 гарантирует разрушение классического решения за конечное время при условии (33). Следовательно, решение из $C^{1}\left(\left[0, T_{0}\right), C^{2}([0, l])\right)$, и поэтому решение из $C^{1}\left(\left[0, T_{0}\right), C([0, l])\right)$ существует лишь для $T_{0} \leqslant T_{1}$. А тогда в силу теоремы 1 имеем

$$
\limsup _{t \uparrow T_{0}}\|A(t)(w(x)(t))\|_{C([0, l])}=+\infty
$$

Из результатов п. 3.5 следует, что оператор $A(t): Z \rightarrow Z$ равномерно по $t$ ограничен на каждом ограниченном множестве пространства $C([0, l])$. Поэтому из $(37)$ имеем

$$
\limsup _{t \uparrow T_{0}}\|w(x)(t)\|_{C([0, l])}=+\infty
$$

а в силу соотношения (22) и неравенств $0 \leqslant t<T_{0}<+\infty$ получаем (36). Теорема доказана.

ЗАмечание 1 . Также из наших рассуждений следует, что в случае $T_{0}=T_{1}$ имеет место более сильное утверждение (35).

\section{ПРИЛОЖЕНИЕ}

\section{ДОКАЗАТЕЛЬСТВО ТЕОРЕМЫ 1}

1. Прежде всего докажем локальную разрешимость задачи Коши (12). Заметим, что следствием задачи (12) является интегральное уравнение

$$
y(t)=y_{0}+\int_{0}^{t} A(\tau)(y(\tau)) d \tau,
$$

или $y=\mathbb{A} y$, где

$$
\mathbb{A} z \equiv y_{0}+\int_{0}^{t} A(\tau)(z(\tau)) d \tau
$$

- оператор, действующий в банаховом пространстве $\mathbb{B}=C([0, T], B)$, а величина $T>0$ будет определена ниже. Интеграл в правой части (38) имеет смысл, поскольку подынтегральное выражение непрерывно в силу условия $y \in C^{1}\left(\left[0, T_{0}\right), B\right)$ и условия 3 теоремы на оператор $A(t)(y)$. Положим также $\|z(t)\|_{\mathbb{B}}=\sup _{t \in[0, T]}\|z(t)\|$. 
Мы докажем локальную разрешимость уравнения (38) в классе $C\left(\left[0, T_{0}\right), B\right)$ и после этого покажем, что каждое его решение является решением задачи (12) из класca $C^{1}\left(\left[0, T_{0}\right), B\right)$.

Заметим, что из (13) и условия 1 теоремы на оператор $A(t)(y)$ следует

$$
\|A(t)(z)\| \leqslant \mu(\|z\|)\|z\| \text {. }
$$

Зафиксируем теперь произвольное число $R>0$ и рассмотрим замкнутое подмножество

$$
\mathbb{B}_{R}=\left\{y(t) \in \mathbb{B} \mid \sup _{t \in[0, T]}\left\|y(t)-y_{0}\right\| \equiv\left\|y-y_{0}\right\|_{\mathbb{B}} \leqslant R\right\}
$$

пространства $\mathbb{B}$. В силу общих свойств метрических пространств множество $\mathbb{B}_{R}$ само является полным метрическим пространством относительно расстояния, порожденного нормой пространства $\mathbb{B}$. Теперь укажем, как выбрать $T>0$ так, чтобы множество было инвариантным относительно оператора $\mathbb{A}$ и этот оператор являлся сжимающим в $\mathbb{B}_{R}$.

Чтобы удовлетворить первому требованию, достаточно заметить, что (с учетом (39) и (40)) верна цепочка неравенств

$$
\begin{aligned}
\left\|\int_{0}^{t} A(\tau)(y(\tau)) d \tau\right\|_{\mathbb{B}} & \equiv \sup _{t \in[0, T]}\left\|\int_{0}^{t} A(\tau)(y(\tau)) d \tau\right\| \leqslant \\
& \leqslant \int_{0}^{T}\|A(\tau)(y(\tau))\| d \tau \leqslant T \sup _{r \in\left[0,\left\|y_{0}\right\|+R\right]} \mu(r) \cdot\left(\left\|y_{0}\right\|+R\right) .
\end{aligned}
$$

Поскольку функция $\mu(\cdot)$ ограничена на любом ограниченном числовом подмножестве в $[0,+\infty)$, можно выбрать такое $T>0$, что правая часть будет меньше $R$ и тем самым первое требование будет выполнено. Теперь обеспечим выполнение второго требования. С учетом (13) и (40) имеем

$$
\begin{gathered}
\left\|\int_{0}^{t} A(\tau)\left(y_{1}(\tau)\right) d \tau-\int_{0}^{t} A(\tau)\left(y_{2}(\tau)\right) d \tau\right\|_{\mathbb{B}} \leqslant \int_{0}^{T}\left\|A(\tau)\left(y_{1}(\tau)\right)-A(\tau)\left(y_{2}(\tau)\right)\right\| d \tau \leqslant \\
\quad \leqslant T \sup _{r \in\left[0,\left\|y_{0}\right\|+R\right]} \mu(r) \cdot \sup _{t \in[0, T]}\left\|y_{1}(t)-y_{2}(t)\right\|=T \sup _{r \in\left[0,\left\|y_{0}\right\|+R\right]} \mu(r) \cdot\left\|y_{1}-y_{2}\right\|_{\mathbb{B} .} .
\end{gathered}
$$

Теперь, если понадобится, мы уменьшим $T$ настолько, чтобы выполнялось условие $T \sup _{r \in\left[0,\left\|y_{0}\right\|+R\right]} \mu(r) \leqslant 1 / 2$. Итак, при выборе $T$ из условий

$$
T \sup _{r \in\left[0,\left\|y_{0}\right\|+R\right]} \mu(r) \cdot\left(\left\|y_{0}\right\|+R\right) \leqslant R, \quad T \sup _{r \in\left[0,\left\|y_{0}\right\|+R\right]} \mu(r) \leqslant \frac{1}{2}
$$

оператор $\mathbb{A}$ является сжимающим отображением из множества $\mathbb{B}_{R}$ в себя.

Следовательно, согласно теореме о неподвижной точке при таком выборе $T$ получаем, что уравнение (38) имеет единственное решение из $\mathbb{B}$. Но тогда с учетом условия 3 на оператор $A$ подынтегральное выражение в (38) непрерывно как композиция непрерывных отображений и, таким образом, $y(t)$ представимо в виде интеграла с переменным верхним пределом от непрерывной функции. Следовательно, $y(t)$ принадлежит классу $C^{1}([0, T], B)$ и является решением задачи Коши (12). Итак, локальная разрешимость задачи (12) доказана. 
Приведенное рассуждение доказывает также единственность решения задачи (12) (независимо от способа его получения), так как, рассмотрев интегральное уравнение (38) с началом в точке, где решение расходится, мы сразу же пришли бы к противоречию с теоремой о неподвижной точке.

2. Перейдем к доказательству альтернативы, сформулированной в теореме. Рассмотрим всевозможные функции $z(t) \in C^{1}([0,+\infty), B)$. Если среди них существует функция, удовлетворяющая (12) при всех $t \geqslant 0$, утверждение теоремы установлено. Если же нет, положим

$$
T_{0} \equiv \sup \left\{T: \exists \text { решение задачи }(12) \text { из } C^{1}([0, T], B)\right\} \text {. }
$$

В силу п. 1 доказательства $T_{0}>0$. В силу доказанной выше единственности возможны два случая: а) существует решение из $C^{1}\left(\left[0, T_{0}\right], B\right) ;$ б) существует лишь решение из $C^{1}\left(\left[0, T_{0}\right), B\right)$.

В случае "а", очевидно, в точке $T_{0}$ снова выполняются все условия теоремы и, следовательно, в силу п. 1 доказательства решение может быть продолжено по $t$ хотя бы на небольшой отрезок. При этом, очевидно, $\lim _{t \uparrow T_{0}} y^{\prime}(t)=\lim _{t \downarrow T_{0}} y^{\prime}(t)=$ $A\left(T_{0}\right)\left(y\left(T_{0}\right)\right)$, поэтому полученное "составное" решение непрерывно дифференцируемо на всем отрезке существования. Это противоречит предположению о непродолжимости решения из $C^{1}([0, T], B)$ за точку $T_{0}$, и, следовательно, случай "а" исключается. В случае "б" соотношение (14) может иметь или не иметь место. Если оно выполнено, утверждение теоремы доказано. В противном случае имеем $\lim \sup _{t \uparrow T_{0}}\|A(y)\|=\mathcal{L}<+\infty$, т. е. вещественная функция $\|A(t)(y(t))\|$, а с нею и $\left\|y^{\prime}(t)\right\|$ (в силу уравнения (12)) ограничены. Но тогда абстрактная функция $y(t)$ липшиц-непрерывна в левой полуокрестности точки $T_{0}$ с константой $\mathcal{L}$ и, следовательно, может быть непрерывно продолжена в точку $T_{0}$ в силу выполнения критерия Коши существования предела функции. Тогда, рассуждая аналогично предыдущему, устанавливаем из интегрального уравнения (38), что и производная $y^{\prime}(t)$ существует и непрерывна вплоть до $T_{0}$ включительно, т. е. мы продолжили решение до $y(t) \in C^{1}\left(\left[0, T_{0}\right], B\right)$ и пришли к случаю "a", невозможность которого установлена ранее. Итак, в случае отсутствия глобального по времени решения возможен лишь случай "б" и при этом должно выполняться предельное соотношение (14). Это и доказывает теорему.

Благодарности. Работа выполнена при поддержке РФФИ (гранты № 11-0112018-офи_м-2011 (М. О. Корпусов) и № 12-01-00479_а (А. А. Панин)) и Министерства образования и науки РФ, соглашение 8215 (М. О. Корпусов).

\section{Список литературы}

[1] T. B. Benjamin, J. L. Bona, J. J. Mahony, Philos. Trans. Roy. Soc. London. Ser. A, 272:1220 (1972), 47-78.

[2] M. O. Korpusov, Nonlinear Anal., 75:4 (2012), 1737-1743.

[3] J.P. Albert, J. Math. Anal. Appl., 141:2 (1989), 527-537.

[4] J. D. Avrin, J. A. Goldstein, Nonlinear Anal., 9:8 (1985), 861-865.

[5] E. Bisognin, V. Bisognin, C. R. Charão, A. F. Pazoto, Port. Math. (N.S.), 60:4 (2003), $473-504$. 
[6] P. Biler, Differ. Integral Equ., 5:4 (1992), 891-901.

[7] Yu. Chen, Appl. Anal., 30:1-3 (1988), 1-15.

[8] N. Hayashi, E. I. Kaikina, P. I. Naumkin, I. A. Shishmarev, Asymptotics for Dissipative Nonlinear Equations, Lecture Notes in Mathematics, 1884, Springer, New York, 2006.

[9] T. Hagen, J. Turi, Comput. Appl. Math., 17:2 (1998), 161-172.

[10] M. Mei, L. Liu, Ya. S. Wong, Nonlinear Anal., 67:8 (2007), 2527-2539.

[11] V. A. Galaktionov, E. L. Mitidieri, S. I. Pohozaev, Nonlinear Anal., 70:8 (2009), 2930-2952.

[12] A. B. Al'shin, M. O. Korpusov, A. G. Sveshnikov, Blow-Up in Nonlinear Sobolev Type Equations, De Gruyter Series in Nonlinear Analysis and Applications, 15, Walter de Gruyter, Berlin, 2011.

[13] Л.К. Эванс, Уравнения с частными производными, Университетская серия, 7, Тамара Рожковская, Новосибирск, 2003.

Поступила в редакцию 27.09.2012 